Meta

Journal des traducteurs

Translators' Journal

\title{
La représentation des connaissances ; son apport à l'étude du processus de traduction
}

\section{Jeanne Dancette et Sonia Halimi}

Volume 50, numéro 2, avril 2005

Processus et cheminements en traduction et interprétation Processes and Pathways in Translation and Interpretation

URI : https://id.erudit.org/iderudit/010999ar

DOI : https://doi.org/10.7202/010999ar

Aller au sommaire du numéro

Éditeur(s)

Les Presses de l'Université de Montréal

ISSN

0026-0452 (imprimé)

1492-1421 (numérique)

Découvrir la revue

Citer cet article

Dancette, J. \& Halimi, S. (2005). La représentation des connaissances ; son apport à l'étude du processus de traduction. Meta, 50(2), 548-559.

https://doi.org/10.7202/010999ar
Résumé de l'article

Cet article fait un survol des études cognitives traitant de l'organisation et de la représentation des connaissances tant pour la lecture que la traduction. Nous postulons que l'enseignement de la traduction vise à amener l'apprenant au niveau de l'expert, que le processus de traduction est perfectible et que la traduction sera améliorée si l'enseignant et l'apprenant ciblent les méthodes et les stratégies inhérentes à la tâche. Parmi les savoir-faire pertinents, nous traitons en fin d'article de la structuration des informations sous forme de carte conceptuelle. 


\title{
La représentation des connaissances; son apport à l'étude du processus de traduction
}

\author{
JEANNE DANCETTE \\ Université de Montréal, Montréal, Canada \\ jeanne.dancette@umontreal.ca
}

SONIA HALIMI

Université de Genève, Genève, Suisse

sonia.halimi@eti.unige.ch

\begin{abstract}
RÉSUMÉ
Cet article fait un survol des études cognitives traitant de l'organisation et de la représentation des connaissances tant pour la lecture que la traduction. Nous postulons que l'enseignement de la traduction vise à amener l'apprenant au niveau de l'expert, que le processus de traduction est perfectible et que la traduction sera améliorée si l'enseignant et l'apprenant ciblent les méthodes et les stratégies inhérentes à la tâche. Parmi les savoir-faire pertinents, nous traitons en fin d'article de la structuration des informations sous forme de carte conceptuelle.
\end{abstract}

\section{ABSTRACT}

This paper is a survey of cognitive studies dealing with the organization and the representation of knowledge in the tasks of both reading and translating, in the field of expertise development. We postulate that the didactics of translation aims to bring the learner to the expert level and that the performance can be improved when methods and strategies are specifically targeted and taught. Among the know-hows pertaining to the task, this paper develops the use of conceptual maps as a means to maximize and structure knowledge and information dealt with in the text.

\section{MOTS-CLÉS/KEYWORDS}

organisation des connaissances, carte conceptuelle, stratégie, enseignement de la traduction

\section{INTRODUCTION}

Dans cet article, nous examinons l'importance de la représentation des connaissances en traduction. Nous avançons l'idée que l'usage des représentations conceptuelles (schémas, cartes conceptuelles) peut faciliter la compréhension et la traduction (lexique, rhétorique discursive) et favoriser l'accumulation du savoir dans les domaines de spécialité.

Selon l'approche cognitive, le processus de traduction se définit essentiellement par le traitement de l'information contenue dans le texte à traduire et par sa mise en relation avec les connaissances antérieures (linguistiques et extralinguistiques). Le traducteur est dans la situation de l'apprenant face à un nouveau texte: "when a learner acquires knowledge from a text, the cognitive representations generated are not those of the text but rather of the objects, states, events, actions and relations represented in the text» (Denhière et Baudet 1991: 159). Le principal apport des sciences cogni- 
tives à l'étude de la lecture, et de la traduction en particulier, tient au rôle de la structuration des connaissances et à la compréhension du mode de construction des référents cognitifs.

Notre réflexion repose sur les observations et interrogations suivantes:

1. La traduction implique non seulement un traitement des unités linguistiques, mais aussi des connaissances. Cela est considéré comme une évidence: "That world knowledge plays an important role in translation and oral interpreting should not be controversial» (Jarvella et al. 2002: 182). Tant le décodage des informations que leur reproduction dans la langue reposent sur la structuration des connaissances, leur emmagasinage et leur réactivation. Mais, paradoxalement, le traitement des connaissances a peu fait l'objet d'études en traductologie, contrairement à l'aspect linguistique de la traduction.

2. La traduction met en œuvre certains processus cognitifs qui ne peuvent pas être pris en charge par la machine. La saisie de l'intention de l'auteur, l'appréciation du contenu et des aspects formels, la réponse aux faits de langue imprévisibles tels que les jeux de mots et les références intra- ou intertextuelles, l'ironie et les sousentendus, etc. relèvent de la psychologie et de l'affectif. Nous avançons toutefois l'idée que la méthode et l'apprentissage de la traduction peuvent être systématisés, formalisés et enseignés.

3. À des fins de recherche, il est important d'identifier, parmi les multiples opérations traduisantes, les activités cognitives qui se prêtent à l'observation et au contrôle empiriques. L'habileté du traducteur à construire et à exploiter ses bases de connaissances semble être une variable sur laquelle on peut mesurer les effets de méthodes et d'outils pédagogiques censés la développer.

Dans cet article, nous donnerons, en premier lieu, un aperçu des travaux sur l'organisation des connaissances en sciences de l'éducation. Ensuite, partant du principe que la traduction est une activité qui nécessite une expertise, nous passerons en revue les travaux de traductologie qui mettent l'accent sur l'apprentissage et les stratégies de traduction, par opposition à une approche traditionnelle. Nous expliquerons, en dernier lieu, comment la représentation des connaissances peut être systématisée et intégrée comme stratégie de traduction et donnerons un exemple tiré de la pratique de l'enseignement de la traduction.

Nous développons l'hypothèse selon laquelle la carte conceptuelle peut, comme outil privilégié de représentation des connaissances, induire des traitements cognitifs spécifiques, indispensables dans le processus de traduction.

\section{L'ORGANISATION DES CONNAISSANCES : L'APPORT DES SCIENCES DE L'ÉDUCATION}

Un des principes fondamentaux de l'approche cognitive est que le savoir ne se reçoit pas, mais que le sujet à qui sont transmises les connaissances le construit. Tout apprentissage serait le fruit d'une démarche active prise en charge par la personne qui acquiert des connaissances et des habiletés. La pédagogie devrait favoriser tant l'acquisition des connaissances que le développement des structures cognitives permettant d'exploiter ces connaissances.

Si l'apprenant est actif et constructif, il doit comprendre son propre processus d'apprentissage et apprendre à mobiliser ses capacités intellectuelles et d'abstraction. 
L'intervention pédagogique consiste alors à lui fournir les outils qui lui permettront de devenir conscient de ses stratégies d'apprentissage, nécessaires pour construire son savoir. Dans cette perspective, Resnick (1984) considère que tout projet pédagogique se définit sur trois dimensions. 1) Sur le plan de l'expertise, le projet vise l'identification et la description des structures de connaissances à développer chez l'apprenant; 2) sur le plan de l'acquisition, le projet s'appuie sur la compréhension $\mathrm{du}$ processus de construction des connaissances; et 3) en matière d'intervention, le projet définit les moyens et actions à prendre par l'enseignant. L'organisation des connaissances ne peut s'intégrer dans un projet pédagogique actif que si ces trois dimensions sont également développées. Nous verrons dans ce qui suit comment cette triade s'applique à la traduction.

\subsection{Développement de l'expertise}

Les études portant sur l'expertise démontrent que la représentation des connaissances n'est pas la même chez les experts et les novices. On reconnaîtrait chez l'expert une organisation des connaissances en structures de type schémas, ou blocs de connaissances, dans lesquelles les concepts sont reliés entre eux par des relations définies. Cette organisation permettrait à l'apprenant de reconnaître les connaissances pertinentes dont il dispose et de les activer rapidement dans les situations qui les rappellent. C'est là que l'expert se distingue du novice: "La différence entre l'efficacité de l'expert et celle du novice réside dans le fait que les connaissances sont bien organisées dans la structure cognitive des premiers alors qu'elles ne le sont pas encore dans celle des derniers» (Tardif 1992: 41).

Ainsi, la performance de l'expert ne s'explique pas seulement par une simple accumulation d'information mais par l'aptitude à organiser ses connaissances en vue d'une meilleure récupération de l'information, "allowing efficient, flexible, and reliable retrieval of information" (Ericsson et Kintsch 1995: 211). Ce serait ce qui caractérise l'expert: "It is a matter of what kind of things one knows and how those things are cognitively arranged, represented, and stored in or retrieved from a knowledge accumulation process that emphasises the development of awareness and familiarity with the nature and structure of problems in the domain» (Shreve 2002: 161). L'organisation des connaissances crée les conditions favorables pour que le sujet prenne conscience des problèmes à résoudre et se les rende familiers (faciles à traiter).

Autrement dit, le système cognitif de l'apprenant n'est pas seulement constitué de connaissances factuelles, mais repose aussi sur des connaissances dynamiques qui orientent l'utilisation des informations qu'il acquiert. Il convient de considérer ces connaissances dynamiques comme des stratégies cognitives puisqu'elles permettent à l'apprenant d'utiliser l'ensemble des ressources dont il dispose pour réaliser efficacement une tâche. C'est la raison pour laquelle ces stratégies doivent être enseignées et présentées explicitement, souligne Tardif (1992).

Cette même idée se retrouve dans la distinction classique entre les connaissances sémantiques - à savoir les connaissances générales, les concepts et propriétés; ainsi que les nouvelles connaissances obtenues lors de la lecture d'un texte -, et les connaissances épisodiques. "[E]pisodic knowledge is a collection of specific job-relevent events or situations (i.e. episodes) that becomes a source for future problem solutions" (Schenk et al. 1998: 15). Les connaissances épisodiques sont stockées dans la mémoire sous 
forme de schémas ou scripts contenant les souvenirs et expériences de la personne. Ces connaissances sont spécifiques aux experts; elles proviennent de l'expérience accumulée pendant des années et sont activées lorsqu'une nouvelle situation analogue est rencontrée. C'est dans cette interaction de plusieurs types de connaissances qu'intervient la procéduralisation et que le mode d'apprentissage peut être déterminant.

Le rôle de l'enseignant serait de fournir à l'apprenant un moyen d'organiser mentalement les connaissances en délimitant le cadre conceptuel du domaine étudié et en identifiant les relations qu'entretiennent entre eux les différents ensembles de concepts. Ce passage de connaissances fragmentées en connaissances par blocs conceptuels se fait facilement après des années de pratique ou en rendant l'apprenant attentif à une méthode de conceptualisation. Anderson (1982) définit la "procéduralisation» comme la «transformation des connaissances déclaratives (qui incluent les connaissances sémantiques et épisodiques) en connaissances procédurales », à savoir les dispositions et procédures pour permettre une action (le comment de l'action). Cette expérience cognitive suppose un processus de prise de décision qui, à son tour, repose sur une démarche logique. En d'autres termes, la procéduralisation permet de convertir les connaissances déclaratives d'un domaine en des règles de production sous forme d'états conditionnels (si-donc) qui déterminent la méthode de résolution de problèmes.

Shreve (2002: 163) applique la logique des règles de production au processus traductif. L'apprenant doit apprendre à reconnaître un schéma particulier dans le texte source (know what it is), ce qu'il implique dans le contexte (identify a domainrelevant meaning), et enfin, définir la manière de résoudre les problèmes pour effectuer la tâche de traduction (know what problem-resolution method to apply). La traduction exige la récupération des connaissances déclaratives et l'application des connaissances procédurales. Cette interaction se fait grâce à la mise en œuvre d'une stratégie appropriée au problème de traduction.

Enfin, notons l'intervention d'une quatrième variable. L'interaction des trois types d'habiletés requiert la concentration, comme le révèlent de manière la plus évidente les études en interprétation. Moser-Mercer $(1997,2000)$ examine le mode d'acquisition de l'expertise chez l'interprète. La concentration est la condition préalable pour la mise en œuvre des savoirs et de la capacité d'anticipation: "The fact that it [concentration] co-varies with a number of other difficulties, such as comprehension, self-monitoring and finding the structure of a speech, indicates that indeed concentration or lack thereof, is the single most important determiner of success or failure in interpreting " (MoserMercer 2000: 349).

\subsection{Acquisition des connaissances et intervention pédagogique (outils)}

Il est admis en psychologie cognitive que les connaissances sur le monde (événements, actions et situations) sont emmagasinées dans la mémoire sous forme de représentations internes organisées en réseaux ou en schémas (Schank et Abelson 1977, Anderson 1982, Lakoff 1987, Schenk et al. 1998). Les recherches empiriques sur la compréhension de textes semblent indiquer que le lecteur construit diverses représentations à partir de la lecture du texte. Kintsch et van Dijk (1983) décrivent trois niveaux: la base de texte (microstructure), la macrostructure (composée des éléments les plus importants du texte) et les modèles de situation (auxquels renvoient 
les événements relatés dans les textes). Johnson-Laird (1983) parle plutôt de modèles mentaux. Ces représentations dans la mémoire sont des structures cognitives qui, par leur caractère de modèles, favorisent la construction graduelle et organisée des connaissances d'un texte à l'autre.

La plupart des études évoquées plus haut concernent la lecture et la compréhension de textes chez les enfants. Nous verrons dans ce qui suit comment les enseignements de telles études s'appliquent à la traduction. La question est de savoir s'il existe des connaissances, des stratégies et des méthodes systématisables et reproductibles dans les situations de traduction nouvelles.

\section{STRATÉGIES DE TRADUCTION}

La plupart des auteurs associent l'activité de la traduction à une démarche de résolution de problèmes spécifique et distinguent entre les stratégies strictement traductionnelles (transfert) et les stratégies procédurales qui tiennent à la méthode et au contrôle de la traduction, en fonction des normes et du mandat reçu (ou perçu). Parmi les travaux portant sur la description des stratégies, mentionnons à titre d'exemples Königs (1996), Jääskeläinen (1993), Tirkkonen-Condit (1989), Lörscher (1991), Dancette (1995), Kiraly (1995), Kussmaul (1995), Wilss (1996), Künzli (2003). Toutefois, on peut constater que les travaux dans le domaine n'ont pas poussé très loin la question de la prise de décision, outre la simple description des instances de raisonnement conduisant à la décision. En conséquence, l'apprentissage des connaissances procédurales est rarement pris en compte dans l'étude du processus de traduction.

Wilss (1996) impute ce désintérêt au fait que les efforts ont initialement porté sur le développement de modèles formels visant à rendre compte de stratégies décisionnelles systématiques et optimales. Un tel objectif est reconnu impossible à atteindre à cause de la complexité inhérente à la démarche traductionnelle. Un modèle formel de prise de décision n'est pas applicable à la traduction, dit-il, sauf dans des situations très limitées ou pour des structures syntaxiques très simples. Wilss propose plutôt de considérer les stratégies de prise de décisions en traduction comme résultant de l'interaction de quatre facteurs: le système cognitif du traducteur, ses connaissances de base, la spécificité du problème et la reconnaissance des procédures de résolution de problème :

All four factors together enable the translator to build up an internal problem representation which, once it has been constructed, will profoundly influence the subsequent decision-making performance of the translator, taking account of «probability ratings », which are an important element in many translation decision-making settings (Wilss 1996: 191).

La résolution du problème tient bien à la conciliation des connaissances déclaratives et des connaissances procédurales. Mais sur quels aspects portent les décisions en traduction? Elles supposent toutes un jugement sur la correction au regard des normes et sur la valeur de l'information - ou au moins sa plausibilité - (Gile 1990), ainsi que l'implication dans le discours. Ces trois facteurs se construisent sur la base des connaissances du domaine. Nous avons de plus postulé que la traduction ne se limite pas à un transfert d'informations, mais implique une construction de connaissances. Pour maximiser les possibilités de l'acquisition de nouvelles connaissances et 
de leur réutilisation, nous avons posé comme essentiel le principe de leur organisation en structures. Dans ce qui suit, nous présentons ce que pourrait apporter à la traduction une démarche basée sur l'organisation des connaissances.

\section{L'ORGANISATION DES CONNAISSANCES, ÉLÉMENT INDISPENSABLE DE LA MÉTHODE DE TRADUCTION}

\subsection{Conceptualisation}

Les textes spécialisés font référence à des concepts qui ont été classés et définis par les experts du domaine. Le lecteur qui ne détient pas le savoir de l'expert doit pouvoir accéder aux connaissances utiles pour comprendre et traduire le texte.

Dans le domaine du droit, Lundquist (1998) analyse les concepts de meurtre, meurtrier, victime et mort tels qu'ils se présentent dans différents textes juridiques en France et en Hollande. Elle montre comment le texte se construit autour du concept clé qui appelle les idées associées; elle décrit l'élaboration chez le lecteur d'une représentation ad hoc, évanescente et non reproductible - le cadre mental - et le passage à une autre, la carte conceptuelle qui serait un produit plus fini, plus durable et plus généralisable. Dans le domaine technique, Lethuillier (2003: 387) met l'accent sur l'identification des réseaux notionnels qu'il est indispensable d'inclure dans l'enseignement de la traduction. Le savoir doit être intégré et réutilisé. Il préconise une «approche conduisant à une optimisation de la démarche d'apprentissage [des concepts] par le recours à des moyens de type levier, dont les effets se cumuleraient et se renforceraient».

Rares sont les recherches, toutefois, qui présentent des résultats empiriques sur la structuration des connaissances en traduction. Les études de protocoles de verbalisation (raisonnement à voie haute) ont ouvert des pistes de recherche qui seraient à poursuivre. Dancette (2003) insiste sur le rôle des référents cognitifs dans la construction d'une image cohérente du texte. Comparant les performances d'étudiants en traduction (allemand-français) de niveaux intermédiaire et avancé, Olohan (2000) étudie le lien entre la conceptualisation du domaine et la qualité des traductions. L'analyse des explicitations et des décisions prises pendant la traduction permet de mesurer l'étendue de la «conscience conceptuelle» des étudiants.

Ces études montrent que le manque d'une connaissance conceptuelle extralinguistique conduit à des imprécisions dans la traduction ou à des hésitations dans le choix des équivalents ou au contraire à des compensations (over-compensation). Elles permettent d'observer et de décrire le phénomène de conceptualisation, mais ne parviennent pas à en définir le mode ni le niveau, ni à le systématiser. La carte conceptuelle faciliterait-elle le processus de conceptualisation et, de là, le processus de traduction? Serait-elle un outil à développer?

\subsection{Carte conceptuelle en traduction}

Dans le cadre d'expériences sur l'acquisition des connaissances, Ausubel (1968) et Novak et Gowin (1984) introduisent la stratégie de la carte conceptuelle comme moyen d'organisation des connaissances en vue d'un apprentissage signifiant (meaningful learning) et de l'intégration de l'information dans les structures cognitives existantes. 
Depuis, l'efficacité de la cartographie conceptuelle a été mise à profit pour de nombreux usages: la compréhension de textes (Reader et Hammond 1994, DeeLucas et Larkin 1995); l'évaluation des programmes de cours en fonction des concepts utilisés dans les livres didactiques (Wu et al. 2004); l'acquisition des connaissances pédagogiques des enseignants (Thomson 1997, Zanting et al. 2003); la recherche documentaire et l'extraction automatique d'information (Debourges et al. 2001), etc. Vu la multiplicité des formes que prend la carte conceptuelle, il convient d'en donner notre définition.

D’un point de vue général, la carte conceptuelle est une représentation graphique des concepts extraits, de manière plus ou moins intuitive, d'un texte ou ensemble de textes. Elle procède d'une réduction et d'une abstraction permettant le passage des phrases du texte aux mots (étiquettes) pointant vers les idées principales, ou selon la finalité de la carte, vers les concepts clés du domaine. Elle est donc la représentation schématique du texte en ses éléments conceptuels constructifs. Elle peut aussi, sous une forme plus élaborée, définir et même coder les relations qu'entretiennent entre eux ces concepts. Ainsi, elle s'appuie sur le sémantisme des mots clés, sur les types de relations logiques entre les concepts (partie-tout, hyperonyme, agent, cause, conséquence, etc.) et sur les marques rhétoriques (charnières logiques) qui permettent de mieux saisir les liens.

Ses usages sont nombreux. Elle peut être utilisée pour l'analyse sémantique d'un texte et pour la représentation de son contenu informationnel, ou pour extraire d'une documentation les réseaux conceptuels définissant les connaissances utiles. Avoir à produire une carte conceptuelle amène l'étudiant à organiser lui-même la structuration de ses connaissances, c'est-à-dire à hiérarchiser les concepts et à les mettre en relation les uns avec les autres, à "échafauder» les connaissances (concept de scaffolding, Eggen et Kauchak 1998). Elle permet à l'étudiant d'articuler ses connaissances préalables avec ses connaissances actuelles, de s'orienter dans un texte ou un domaine; et de s'approprier et retenir les connaissances. En tant qu'élément d'une stratégie d'enseignement, la carte conceptuelle est un structurant cognitif. On peut donc la considérer comme une technique pour réguler les processus cognitifs.

En traduction, la carte conceptuelle peut être appliquée de façon avantageuse à deux niveaux: celui du texte à traduire et celui de la documentation d'appui. Elle vise à stimuler l'interrogation conceptuelle qui, selon nous, doit faire partie du processus de la traduction. En interprétation consécutive, Nguyen (1998) et Nguyen et Tochon (1995) comparent la performance de trois groupes d'interprètes, selon qu'ils utilisent 1) la carte conceptuelle établie par le professeur/expérimentateur et mise à la disposition des sujets lors de l'interprétation; 2) le résumé du texte fourni par le professeur/ expérimentateur; 3) aucun outil. L'application de deux techniques d'intervention la carte conceptuelle et le résumé - démontre une influence positive de la carte conceptuelle et négative du résumé sur les quatre variables impliquées dans ce processus: la compréhension, la production, la prise de notes et l'état psychologique.

Nous donnons, à la fin de cet article, un exemple de carte conceptuelle appliquée à un texte anglais à traduire en français afin d'illustrer le genre d'interrogation conceptuelle qu'un texte doit/peut engendrer. 


\subsection{Exemple d'application en traduction}

Le texte ci-dessous, représentatif de nombreux écrits mi-techniques mi-vulgarisés, établit un réseau de liens entre concepts de manière pas toujours explicite. Nous voulons démontrer que les cartes ne sont pas seulement un moyen de visualisation mais aussi un «outil pour l'établissement d'un ordre d'exécution» (Lethuillier 2003: 387).

\section{DIAMONDS ARE FOREVER}

Along with the almost frantic interest in gem diamonds has come an unprecedented proliferation of uses for the lesser-quality diamonds that literally make our industrial system work. Grinding, sawing, drilling, and polishing are the oldest and most common tasks, but diamonds are proving indispensable in exotic jobs as well:

- In hospitals around the world, eye surgeons can now remove cataracts with supersharp diamond knives whose edges are so even that no imperfections are visible at a thousand times magnification.

- A pinhead-size gold-coated diamond cube is an essential element in high-capacity miniature transmitters that carry television and telephone signals across the United States. Since diamonds have the greatest thermal conductivity of any material, they keep the tiny transmitters from burning up.

Diamond values are determined by carat weight, cut, clarity, and color. Carat is an ancient term referring to the uniform weight of a carob seed.

(Les termes en gras sont les concepts gravitant autour du concept clé diamond; le soulignement indique les passages où la traduction pouvait être facilitée par une représentation des notions.)

L'élaboration de la carte, cette transformation du texte (ensemble de phrases) en ses éléments constitutifs et leur réduction en concepts clés, est illustrée par les figures 1 et 2 .

FIGURE 1

Informations sur le concept «Diamond» induites du texte

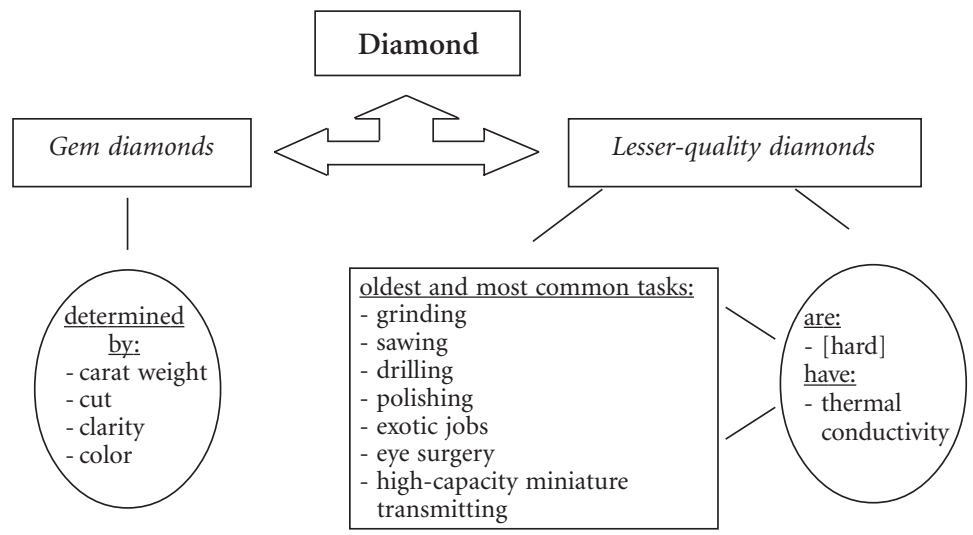

Le texte énonce les faits suivants: on distingue deux types de diamants =>ils ont des propriétés définissant chaque type $=>$ ils se prêtent à des utilisations propres à chaque type $=>$ certaines utilisations sont traditionnelles, d'autres récentes. 
Ce résumé se trouve schématiquement représenté par la figure 1. C’est par un procédé de paraphrasage du texte qu'il a pu être établi. Les paraphrases sont une étape constitutive de la représentation conceptuelle. La figure 2 est l'étape suivante: elle explicite les relations qui lient les concepts entre eux dans le texte (et la documentation).

FIGURE 2

Représentation correspondante en français et spécification des liens

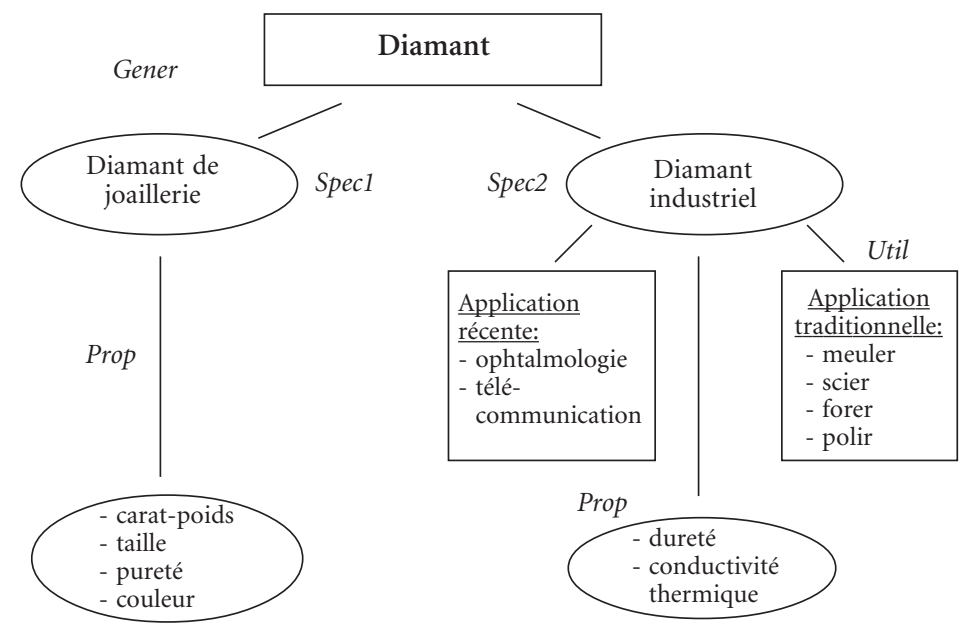

Cette figure utilise des catégories plus générales que la première en nommant les liens (spécifique, propriété, utilisation) et en utilisant une terminologie validée. Ainsi les liens entre gem diamond, lesser quality diamonds et diamond sont-ils notés comme deux co-hyponymes de «diamant»: diamant de joaillerie et diamant industriel. Tasks et jobs sont transcrits au moyen de l'étiquette Util (pour utilisation). Les domaines d'utilisation sont explicités: eye surgery devient ophtalmologie et miniature transmitters that carry television and telephone signals devient télécommunications.

La figure 2 est le résultat de plusieurs opérations cognitives très pertinentes pour la traduction. La transformation du texte en unités de connaissances organisées, d'une part, et d'autre part, l'étiquetage des unités de connaissances plus générales qui font appel à la terminologie du domaine (avec l'appui, si nécessaire, de la documentation et des dictionnaires). Le texte à traduire ainsi travaillé devient le matériau pour l'acquisition de connaissances généralisables et réutilisables à propos d'autres textes du même domaine.

L'apprentissage de la structuration des informations et des connaissances en carte conceptuelle est un élément des pédagogies actives de la traduction, surtout lorsque les textes ont un contenu référentiel important et font appel à des notions peu connues (texte technique) et lorsque la structure argumentative est peu marquée. Dans ce cas, une représentation des champs notionnels facilitera le choix des charnières logiques appropriées. 


\section{CONCLUSION}

Le survol que nous avons présenté a mis en évidence les mécanismes des connaissances procédurales (résolutions de problèmes, stratégies) comme facteurs favorisant l'organisation des connaissances. Nous avons vu que les recherches en éducation ont démontré la pertinence de l'organisation des connaissances. Les pédagogies fondées sur les stratégies sont favorables à une démarche cognitive qui associe l'acquisition des connaissances et le développement des structures cognitives. Le rôle des stratégies étant déterminant en traduction, c'est à ce niveau que nous pensons que l'organisation des connaissances est bénéfique, à court et à long terme, comme élément de la méthode de traduction.

La carte conceptuelle est un outil privilégié favorisant le processus de la traduction. Elle permet d'optimiser l'apprentissage des systèmes notionnels propres à un domaine de spécialité. Elle oriente la réflexion conceptuelle et permet de justifier les choix terminologiques et de mieux comprendre l'organicité textuelle exprimant les rapports entre notions; enfin, elle facilite l'accumulation des connaissances et leur réutilisation à l'occasion d'autres traductions dans le même domaine. Nous ne saurions trop insister sur l'idée que l'usage des connaissances n'est pas fortuit mais optimisable, y compris et surtout dans les périodes d'apprentissage au moyen de méthodes appropriées.

\section{RÉFÉRENCES}

Anderson, J. R. (1982): «Acquisition of Cognitive Skill», Psychological Review 89, p. 369-406.

Ausubel, D.P. (1968): Educational Psychology. A Cognitive View, Holt, Rinehart and Winston, Inc., New York.

Barth, B.-M. (1987): L'apprentissage de l'abstraction. Méthode pour une meilleure réussite de l'école, Retz, Paris.

DAnCette, J. (2003) : «Sens, cohérence et réseaux conceptuels», TTR - Traduction, Terminologie et Rédaction 16-1.

Dancette, J. (1995) : Parcours de traduction; étude expérimentale du processus de compréhension, Presses universitaires de Lille, Lille.

Debourges et al. (2001): "Cartographie de textes: une aide à l'utilisateur dans le cadre de la découverte de nouveaux domaines", Actes TALN 2001, Tours.

Dee-Lucas, D., et J. H. Larkin (1995): «Learning from Electronic Texts: Effects of Interactive Overviews for Information Access», Cognition and Instruction 13-3, p. 431-468.

Denhière, G., et S. BAudet (1992): Lecture, compréhension de texte et science cognitive, Paris, Presses Universitaires de France.

Eggen, P. and D. Kauchak (1998): Educational Psychology, London, Sydney, Toronto, PrenticeHall.

Ericsson, K., et W. Kintsch (1995) : «Long-Term Working Memory», Psychological Review 102, p. 211-245.

GiLE, D. (1990): «La traduction et l'interprétation comme révélateurs des mécanismes de production et de compréhension de discours», Meta 35-1, p. 20-30.

JäÄskeläInen, R. (1993) : «Recent Trends in Empirical Translation Research», in S. TirkKonenCondit and J. Laffling (eds.), Kielitieteellisiäa Tutkimuksia/Studies in Languages. Joensuu, University of Joensuu, Faculty of Arts, p. 99-120.

Jarvella, J. R., et al. (2002): "Towards Characterizing Translator Expertise, Knowledge and Know-How: Some Findings Using TAPs and Experimental Methods» in A. Riccardi (eds.), Translation Studies. Perspectives on an Emerging Discipline, Cambridge University Press, Cambridge, UK. 
Johnson-Laird, P. (1983): Mental Models: Towards a Cognitive science of Language, Inference and Consciousness. Cambridge University Press, Cambridge.

Kintsch, W. (1987): «The Role of Knowledge in Discourse Comprehension: A ConstructionIntegration Model», in Psychological Review 95-2.

Kintsch, W. (1998) : Comprehension; A Paradigm for Cognition, Cambridge, Cambridge University Press.

Kintsch, W. et T. A. Van Dijk (1983): Strategies of Discourse Comprehension, New York, Academic Press.

Kiraly, D. (1995): Pathways to Translation, Pedagogy and Process, Kent State University Press, Kent, Ohio \& London, England.

Königs, F (1996): «Processus mentaux étudiés chez des sujets allemands», Meta 40-1, p. 7-23.

KüNZLI, A. (2003): Quelques stratégies et principes en traduction technique français-allemand et français-suédois, Thèse présentée à l'Université de Stockholm.

Kussmaul, P. (1995): Training The Translator, John Benjamins, Amsterdam/Philadelphia.

Lakoff, G. (1987): Women, Fire and Dangerous Things. What Categories Reveal about the Mind, University of Chicago Press, Chicago/London.

Lethuillier, J. (2003): "L'enseignement des langues de spécialité comme préparation à la traduction spécialisée», Meta 48-3, p. 379-392.

Lörscher, W. (1991): Translation Performance, Translation Process and Translation Strategies, Gunter Narr Verlang, Tübingen.

Lundquist, L. (1998): "Cadres et espaces mentaux dans la structuration cognitive des connaissances - et des discours juridiques", in Y. GAMBiER (ed.) Discours professionnels en français, Peter Lang, Frankfurt.

Moser-Mercer, B., Frauenfelder, U., Casado, B., and A. Künzli (2000) : «Searching to Define Expertise in Interpreting», in B. Englund Dimitrova and K. Hyltenstam (eds.), Language Processing and Interpreting. Proceedings of the International Symposium on Language Processing and Interpreting, Stockholm University, February 1997. John Benjamins, Amsterdam.

Moser-Mercer, B., (2000): "The Rocky Road to Expertise in Interpreting», in Kadric, M., Kaindl, K. \& Pöchhacker, F (eds.) Translationswissenschaft. Festschrift für Mary SnellHornby zum 60. Stauffenburg, Tübingen, p. 339-352

Moser-Mercer, B., (1997): "The Expert-Novice Paradigm in Interpreting Research", in E. Fleischmann et al. (eds.) Translationsdidaktik. Grundfragen der Uberstzungswissenschaft, Gunter Narr, Tübingen, p. 255-261.

Nguyen, Phuong T. C. (1998): Cartes conceptuelles et processus de compréhension en interprétation, thèse présentée à l'Université de Montréal.

Nguyen, Phuong T. C. et F.V. Tochon (1995): Influence de la carte de concepts sur les processus de compréhension et de production orales propres à l'interprétation consécutive, une nouvelle orientation de recherche, UNIFE, Puente, Lima, Pérou.

NovaK, J.D. and D.B. Gowin (1984): Learning How to Learn. Cambridge University Press, Cambridge.

Olohan, M. (2000): «Investigating Domain Conceptualisation and Scene Construal in Trainee Translators ", Babel 48-4, p. 593-606.

Reader, W. R., and Hammond, N. V. (1994) : «Computer-Based Tools to Support Learning from Hypertext: Concept Mapping Tools and Beyond», Computers and Education, 22-1/2, p. 99106.

Resnick, L. B. (1984): «Comprehending and Learning: Implications for a Cognitive Theory of Instruction ", in M. et al., (eds.), Learning and Comprehension, Laurence Erlbaum Associates, Hillsdale, NI.

Schank, R. C., et R.P. Abelson (1977): Scripts, Plans, Goals and Understanding, Lawrence Erlbaum, Hillsdale NJ.

Schenk, K. D., et al. (1998): «Differences Between Novice and Expert Systems Analysts: What Do We Know and What Do We Do?» Journal of Management Information Systems, 15-1, p. 9-50. 
Shreve, G. M. (2002) : «Knowing Translation: Cognitive and Experimental Aspects of Translation Expertise from the Perspective of Expertise Studies », in A. Riccardi (eds.), Translation Studies. Cambridge University Press, Cambridge, UK.

TARdif, J. (1992): Pour un enseignement stratégique. L'apport de la psychologie cognitive, Les Éditions Logiques, Montréal.

Thomson C. (1997): «Concept Mapping as a Means of Evaluating Primary School Technology Programmes", International Journal of Technology and Design Education 7, p. 97-110.

Tirkkonen-Condit, S. and Condit, S. (Eds.) (1989): Empirical Studies in Translation and Linguistics. monistuskeskus, Joensuu, Finland, Joensunn yliopisto.

WiLss, W. (1996): Knowledge and Skills in Translator Behavior, John Benjamins Publishing Company, Amsterdam/Philadelphia.

Wu C-C. et al. (2004) : «Using Concept Maps to Aid Analysis of Concept Presentation in High School Computer Textbooks", Education and Information Technologies 9-2, p. 185-197.

Zanting A. et al. (2003) : «Using Interviews and Concept Maps to Access Mentor Teachers Practical Knowledge», Higher Education 46, p. 195-214. 